\title{
Epidemiologia da Transmissão da Dengue
}

H.M. YANG ${ }^{1}$, Departamento de Matemática Aplicada, Instituto de Matemática, Estatística e Computação Científica, UNICAMP, Cx.P. 6065, 13081-970 Campinas, SP, Brasil.

Resumo. Estuda-se a transmissão do vírus da dengue na população humana através de modelo matemático. Como o vírus é transmitido pelo mosquito Aedes aegypti, o modelo considera a interação entre duas populações.

\section{Introdução}

O primeiro relato, porém não confirmado, da dengue no Brasil data de $1923 \mathrm{em}$ Niterói (RJ) e o primeiro surto epidêmico documentado ocorreu em Boa Vista (RR). A dengue possui somente um ciclo epidemiológico (urbano) que tem como principais elos o homem (hospedeiro) e o mosquito Aedes aegypti (vetor). De um modo geral, as arboviroses predominam nos trópicos, porquanto aí existem condições climáticas mais favoráveis para a propagação contínua dos arbovírus [3].

A fêmea do mosquito, suscetível, infecta-se com o vírus da dengue quando se alimenta de um indivíduo infectante (no período de viremia). Após o período de incubação extrínseca, que vai desde a ingestão do sangue infectado até o momento em que é capaz de transmitir o vírus pela sua replicação nas glândulas salivares, o mosquito permanece infectante até a sua morte, sem nada sofrer ou apresentar lesões mínimas. Este período pode variar de 7 a 10 dias. Quando um mosquito infectante injeta vírus da dengue no hospedeiro suscetível durante o repasto sanguíneo, após um período de incubação que varia, em média, de 4 a 6 dias (mínimo de 3 e máximo de 10 dias), a dengue pode evoluir para forma assintomática, forma clássica com febre, mialgias e artralgias, e para forma grave, conhecida como "dengue hemorrágica", que cursa com distúrbios da coagulação e choque, podendo levar à morte. A duração dos sintomas varia usualmente de 3 a 7 dias e o período infeccioso (viremia) dura apenas alguns dias, variando de 3 a 7 dias. Posteriormente, o indivíduo desenvolve imunidade específica de longa duração [3].

Aceita-se a existência de 4 sorotipos distintos de dengue (sorotipos 1, 2, 3 e 4). Como apresentam baixa imunidade cruzada, ocorrem as chamadas "infecções secundárias" após a primeira infecção por um determinado sorotipo. Mostrou-se que indivíduos infectados com um sorotipo de dengue eram suscetíveis à infecção por outro sorotipo seis meses após a primeira exposição. Entretanto, não há evidência de

\footnotetext{
${ }^{1}$ hyunyang@ime.unicamp.br; apoio financeiro FAPESP e CNPq.
} 
que possa ocorrer uma reinfecção pelo mesmo sorotipo (induzindo a uma imunidade perene). É aceito que a infecção primária por um dos vírus da dengue ocasionaria apenas o quadro clássico da virose. Porém, ao ocorrer uma reinfecção por outro tipo antigênico de vírus dengue, em um intervalo de tempo inferior a 5 anos, seria então desencadeado o quadro hemorrágico da dengue. É admitido que todos os 4 sorotipos de dengue podem provocar quadros hemorrágicos. Porém, estudos clínicos e virológicos evidenciaram uma maior correlação entre os casos de dengue hemorrágica e o sorotipo 2, quando este ocorria como "infecção secundária". Entretanto, há relatos de epidemias de dengue hemorrágica causadas pelo sorotipo 3 , indicando que ou existem cepas virulentas que causam epidemia de dengue hemorrágica ou uma "infecção secundária" pode provocar uma resposta mais intensa, com sintomas de dengue hemorrágica. Outro aspecto epidemiológico interessante é que, ao contrário do que sucede com a maioria das doenças infecciosas, um bom estado nutricional parece ser mais um fator de risco no desenvolvimento da dengue hemorrágica [3]. No Estado de São Paulo já circulou vírus dengue sorotipos 1 e 2, e houve isolamento do sorotipo 3 na Cidade de Campinas (caso importado de outro país).

No Brasil, ao encontrar condições favoráveis, a transmissão da dengue tornou-se um problema de saúde pública em nível nacional e tem sido registrada anualmente desde 1986, com crescente expansão da sua área de ocorrência, atingindo no ano de 1998 a cifra de 537.507 casos distribuídos em 24 Estados, sendo que em 9 destes foi constatada a ocorrência de 98 casos de dengue hemorrágica. No Estado de São Paulo, neste mesmo ano, foram computados 10.629 casos em 102 Municípios, a despeito do crescente envolvimento do poder público municipal e da população no controle do mosquito vetor, o Aedes aegypti, única forma de controle da dengue, uma vez que a vacina ainda não está disponível. As autoridades de saúde pública, por meio de ações de diversos organismos, conseguiram manter a doença em níveis baixos, porém, nos últimos tempos, a prevalência da dengue tem aumentado e atraído a atenção de muitos pesquisadores, inclusive do próprio Ministro da Saúde. Diversos são os fatores que têm contribuído para a reemergência da dengue no Brasil nos últimos tempos, que vão desde a deterioração das condições sociais e o descaso dos serviços públicos até o fenômeno climático "El Niño".

Desenvolve-se um modelo matemático para descrever a dinâmica da transmissão da dengue. O estudo da dinâmica da população de mosquitos [5], que analisou os efeitos da introdução de várias formas de controle, é acoplado à dinâmica da transmissão da dengue na população humana.

\section{Dinâmica da transmissão da dengue}

Estuda-se o caso da circulação de um único sorotipo na comunidade. A dengue é causada por um arbovírus, cuja infeç̧ão nos mosquitos parece não encurtar a vida média e nem criar imunidade. Entretanto, na população humana, esta infecção (quando isolada), que resulta em uma indução de imunidade perene, é uma doença benigna, e os indivíduos sintomáticos desta doença não são levados à morte. A dinâmica da população de mosquitos é aquela apresentada em [5], sendo a po- 
pulação humana subdividida, baseada na história natural da infecção, em indivíduos suscetível, exposto, infectante e recuperado, designados, respectivamente, por $\mathrm{S}-\mathrm{H}$ $I-R$. A transmissão do vírus da dengue considera duas populações.

População de mosquito. A população de mosquitos adultos é subdividida em mosquitos suscetíveis $\left(W_{1}\right)$, infectados porém não infectantes $\left(W_{2}\right)$ e infectantes $\left(W_{3}\right)$. Os mosquitos infectados e não infectantes são retirados a uma taxa $\gamma_{w}$, onde $\gamma_{w}^{-1}$ é o período de incubação extrínseca do vírus da dengue no mosquito. $\mathrm{O}$ número total de mosquitos é $W=W_{1}+W_{2}+W_{3}$. Matematicamente, os mosquitos suscetíveis $\left(W_{1}\right)$ são infectados pela presença de indivíduos infectantes $(I)$, a uma taxa de ataque (força de infeç̧ão) $\eta_{w}$ que depende de $I$, ou seja, $\eta_{w}(I)$, que leva em consideração a taxa de contato entre indivíduos infectantes e mosquitos suscetíveis, designada por $\beta_{w}$. Biologicamente, os mosquitos suscetíveis são infectados quando picam indivíduos infectantes, e a taxa $\beta_{w}$ leva em consideração a freqüência de picadas. Os mosquitos infectados e não infectantes $\left(W_{2}\right)$ e infectantes $\left(W_{3}\right)$ são, em geral, mais velhos, por isso acrescenta-se taxas de mortalidade adicionais devido ao envelhecimento, dados, respectivamente, por $\mu_{2}$ e $\mu_{3}$, com $\mu_{2}<\mu_{3}$. Considera-se mecanismos de controle atuando na população de mosquitos [5].

População humana. A população humana é subdividida em quatro compartimentos não-interceptantes. Matematicamente, os indivíduos suscetíveis $(S)$ são infectados pelos mosquitos infectantes $\left(W_{3}\right)$ e passam para a classe dos expostos $(H)$ a uma taxa de ataque (força de infecção) $\eta_{h}$ que depende de $W_{3}$, ou seja, $\eta_{h}\left(W_{3}\right)$, que leva em consideração a taxa de contato entre mosquitos infectantes e indivíduos suscetíveis, designada por $\beta_{h}$. Biologicamente, os indivíduos suscetíveis são infectados pelas picadas de mosquitos infectantes, aqueles que haviam previamente picado indivíduos infectantes, e a taxa $\beta_{h}$ leva em consideração a freqüência de picadas. Os indivíduos expostos são retirados deste compartimento a uma taxa $\gamma_{h}$, onde $\gamma_{h}^{-1}$ é o período de incubação do vírus da dengue no indivíduo humano. Finalmente, os indivíduos infectantes são retirados deste compartimente a uma taxa $\sigma_{h}$, onde $\sigma_{h}^{-1}$ é o período de infecção (ou recuperação) do indivíduo humano. Não se consideram a perda de imunidade e a presença de anticorpos maternos. A população toda é dada por $N=S+H+I+R$ e todos os indivíduos estão sob a influência de uma mesma taxa de mortalidade dada por $\mu_{h}$ e não há mortalidade adicional pela doença, uma vez que está-se considerando infecção por um único sorotipo.

A dinâmica de transmissão da dengue envolve acoplamento de duas populações. A população de mosquitos, quando há transmissão de dengue, é descrita por

$$
\left\{\begin{array}{llll}
\frac{d}{d t} E(t) & =\varphi(W)\left[1-\frac{E(t)}{C^{\prime}}\right]-\rho_{e} E(t), & & \rho_{e}=\sigma_{e}+\mu_{e}+m_{e}, \\
\frac{d}{d t} L(t) & =\sigma_{e} E(t)-\rho_{l} L(t), & & \rho_{l}=\sigma_{l}+\mu_{l}+\mu_{l}^{\prime}+m_{l}, \\
\frac{d}{d t} P(t) & =\sigma_{l} L(t)-\rho_{p} P(t), & & \rho_{p}=\sigma_{p}+\mu_{p}+\mu_{p}^{\prime}+m_{p}, \\
\frac{d}{d t} W_{1}(t) & =\sigma_{p} P(t)-\left[\eta_{w}(I)+\rho_{w}\right] W_{1}(t), & \rho_{w}=\mu_{w}+\mu_{w}^{\prime}, \\
\frac{d}{d t} W_{2}(t)=\eta_{w}(I) W_{1}(t)-\rho_{2} W_{2}(t), & & \rho_{2}=\gamma_{w}+\mu_{w}+\mu_{w}^{\prime}+\mu_{2}, \\
\frac{d}{d t} W_{3}(t) & =\gamma_{w} W_{2}(t)-\rho_{3} W_{3}(t), & & \rho_{3}=\mu_{w}+\mu_{w}^{\prime}+\mu_{3},
\end{array}\right.
$$

onde $\rho_{e}, \rho_{l}, \rho_{p}, \rho_{w}, \rho_{2}$ e $\rho_{2}$ são, respectivamente, as taxas globais de saída das fases ovo, larva, pupa e mosquitos adultos suscetíveis, latentes e infectantes. Suscintamente, os parâmetros $\sigma_{e}, \sigma_{l}$ e $\sigma_{p}$ são as taxas de transição de uma fase para 
outra do ciclo de vida; $\mu_{e}, \mu_{l}, \mu_{p}$ e $\mu_{w}$ são as taxas de mortalidade natural em cada fase; $\mu_{l}^{\prime}, \mu_{p}^{\prime}$ e $\mu_{w}^{\prime}$ são as taxas de mortalidade adicionais devido ao controle químico; $m_{e}, m_{l}$ e $m_{p}$ são taxas de mortalidade devido ao controle mecânico e $C^{\prime}$ é a capacidade remanescente de criadouros. Descrições detalhadas das taxas podem ser encontradas em [5]. Se $\beta_{w}=0$, isto é, população de mosquitos sem dengue (neste caso, $W=W_{1}$ e $W_{2}=W_{3}=0$ ), então tem-se o modelo descrito em [5].

A população humana, por sua vez, é descrita pelo sistema de equações

$$
\left\{\begin{aligned}
\frac{d}{d t} S(t) & =\mu_{h} N-\left[\eta_{h}\left(W_{3}\right)+\mu_{h}\right] S(t), & & \\
\frac{d}{d t} H(t) & =\eta_{h}\left(W_{3}\right) S(t)-\rho_{h} H(t), & & \rho_{h}=\gamma_{h}+\mu_{h}, \\
\frac{d}{d t} I(t) & =\gamma_{h} H(t)-\rho_{i} I(t), & & \rho_{i}=\sigma_{h}+\mu_{h}, \\
\frac{d}{d t} R(t) & =\sigma_{h} I(t)-\mu_{h} R(t), & &
\end{aligned}\right.
$$

onde $\rho_{h}$ e $\rho_{i}$ são, respectivamente, as taxas globais de saída do compartimento dos indivíduos latentes e infectantes. A efetiva transmissão da dengue ocorre quando um mesmo mosquito picar sucessivamente um indivíduo infectante e, após um período de tempo $\gamma_{w}^{-1}$, picar um indivíduo suscetível.

A duração de uma geração na população de mosquitos é de alguns dias, sendo no máximo seis meses, por isso $W_{3}$ varia no tempo. Comparativamente, a duração de uma geração entre os indivíduos humanos é de dezenas de anos. Por isso, está-se trabalhando com uma população humana constante, fazendo-se a taxa de natalidade igual à taxa de mortalidade, isto é, as mortes são repostas por recém-nascidos [4].

Assim, o sistema de equações (2.2) pode ser dividido pela população constante $N$, e ser escrita em termos de frações de indivíduos $s, h, i$ e $r$, dos compartimentos, respectivamente, de suscetíveis, expostos, infectantes e recuperados, ou seja,

$$
\left\{\begin{aligned}
\frac{d}{d t} s(t) & =\mu_{h}-\left[\eta_{h}\left(W_{3}\right)+\mu_{h}\right] s(t) \\
\frac{d}{d t} h(t) & =\eta_{h}\left(W_{3}\right) s(t)-\rho_{h} h(t) \\
\frac{d}{d t} i(t) & =\gamma_{h} h(t)-\rho_{i} i(t) \\
\frac{d}{d t} r(t) & =\sigma_{h} i(t)-\mu_{h} r(t)
\end{aligned}\right.
$$

Este sistema é acoplado ao sistema de equações (2.1) para descrever a dinâmica da transmissão do vírus da dengue na população humana.

\section{Análise do modelo - Encontro entre indivíduos suscetiveis e infectantes}

Os sistemas de equações (2.1) e (2.3) dependem das forças de infecção $\eta_{w}(I)$ e $\eta_{h}\left(W_{3}\right)$. Para elas, faz-se duas suposições, as mais simples possíveis, obtendo duas formas distintas para descrever a dinâmica da transmissão da dengue

Primeiro, supõe-se que a transmissão da infecção pelos infectantes (homem ou mosquito) para os suscetíveis (mosquito ou homem) seja um evento probabilístico. Nesta situação a relação entre as forças de infecção e densidades (taxas) de picadas $\beta_{w}^{\prime}$ e $\beta_{h}^{\prime}$, constantes, dadas por

$$
\left\{\begin{array}{l}
\eta_{w}(I)=\varepsilon \beta_{w}^{\prime} \frac{I}{N}, \\
\eta_{h}\left(W_{3}\right)=\varepsilon \beta_{h}^{\prime} \frac{W_{3}}{W},
\end{array}\right.
$$


depende da fração da população considerada. Aqui, pode-se entender, em primeira ordem de aproximação, a fração de indivídios infectantes $i \equiv I / N$ como sendo a probabilidade de um mosquito suscetível encontrar (e picar) um indivíduo infectante, e a fração de mosquitos infectantes $w_{3} \equiv W_{3} / W$ como sendo a probabilidade de um indivíduo suscetível ser encontrado (e picado) por um mosquito infectante.

Segundo, supõe-se que a infecção seja transmitida por um encontro aleatório entre os infectantes (homem ou mosquito) e os suscetíveis (mosquito ou homem), isto é, utiliza-se o encontro das massas. Nesta situação, a relação entre as forças de infecção e taxas de contato per-capitas $\beta_{w}$ e $\beta_{h}$, constantes, dadas por

$$
\left\{\begin{array}{l}
\eta_{w}(I)=\varepsilon \beta_{w} I \\
\eta_{h}\left(W_{3}\right)=\varepsilon \beta_{h} W_{3}
\end{array}\right.
$$

depende da quantidade da população considerada.

O parâmetro $\varepsilon$ representa, nas equações (3.1) e (3.2), a forma como os indivíduos estão distribuídos geograficamente, facilitando ou não a transmissão da dengue. As equações (3.1) e (3.2) assumem interpretações biológicas diferentes; no entanto, matematicamente, elas podem representar uma mesma hipótese de quantificação se interpretar $\beta_{w}^{\prime}$ e $\beta_{h}^{\prime}$ como taxas de contato totais, ou sejam, $\beta_{w}^{\prime}=\beta_{w} N$ e $\beta_{h}^{\prime}=\beta_{h} W_{3}$. Neste caso, como $W_{3}$ varia com o tempo, a taxa $\beta_{h}^{\prime}$ também dependeria do tempo, diferente da equação (3.1) que a considera constante. Contudo, esta identificação torna-se ainda mais indiscriminada (matematicamente) se a população toda mantiver-se contante no tempo, ou em equilíbrio estacionário.

O objetivo é estudar a dinâmica da transmissão da dengue e os efeitos dos diversos mecanismos de controle aplicados aos mosquitos. Os resultados detalhados são mostrados para o caso $\varphi(W)=\phi W$, a capacidade de oviposição do mosquito. Os resultados dos outras formas de oviposição [5] podem ser obtidos facilmente.

\subsection{Evento probabilístico}

Estuda-se o caso do evento probabilístico, com as forças de infecções dadas pela equação (3.1). Os pontos de equilíbrio dos sistemas de equações (2.1) e (2.3) com as forças de infecção dadas pela equação (3.1) são três.

População humana livre de mosquitos. Os valores para cada compartimento de mosquitos e homens são dados por

$$
\left\{\begin{array}{c}
E=L=P=W_{1}=W_{2}=W_{3}=0 \\
s=1 \quad \text { e } h=i=r=0
\end{array}\right.
$$

que são válidos, biologicamente, para $\phi<\phi_{t h}$ e $R<1$, onde a razão de reprodutibilidade da dengue $R$ é dada por

$$
R=\frac{\gamma_{w} \gamma_{h} \varepsilon^{2} \beta_{w}^{\prime} \beta_{h}^{\prime}}{\rho_{2} \rho_{3} \rho_{h} \rho_{i}}=\frac{\varepsilon \beta_{h}^{\prime}}{\rho_{3}} \times \frac{\gamma_{h}}{\rho_{h}} \times \frac{\varepsilon \beta_{w}^{\prime}}{\rho_{i}} \times \frac{\gamma_{w}}{\rho_{2}},
$$

$\operatorname{com} \phi_{t h}$, a taxa de oviposição per-capita limiar, sendo dada por

$$
\phi_{t h}=\left(\frac{Q}{\rho_{e}}\right)^{-1}=\left(\frac{\sigma_{e}}{\rho_{e}} \frac{\sigma_{l}}{\rho_{l}} \frac{\sigma_{p}}{\rho_{p}} \frac{1}{\rho_{w}}\right)^{-1} \text {. }
$$


Quando não houver controle no vetor, $R=R_{0}$ é a razão de reprodutibilidade basal.

População humana infestada por mosquitos sem a transmissão da dengue. Os valores para cada compartimento de mosquitos e homens são dados por

$$
\left\{\begin{array}{l}
E=C^{\prime}\left(1-\frac{\phi_{t h}}{\phi}\right) \\
L=\frac{\sigma_{e}}{\rho_{l}} E \\
P=\frac{\sigma_{e}}{\rho_{l}} \frac{\sigma_{l}}{\rho_{p}} E \\
W_{1}=W=\frac{\sigma_{e}}{\rho_{l}} \frac{\sigma_{l}}{\rho_{p}} \frac{\sigma_{p}}{\rho_{w}} E \\
W_{2}=W_{3}=0 \\
s=1 \\
h=i=r=0
\end{array}\right.
$$

que são válidos, biologicamente, para $\phi>\phi_{t h}$ e $R<1$.

População humana infestada por mosquitos com a transmissão da dengue. Os valores para cada compartimento de mosquitos e homens são dados por

$$
\left\{\begin{array}{l}
L=\frac{\sigma_{e}}{\rho_{l}} E \\
P=\frac{\sigma_{e}}{\rho_{l}} \frac{\sigma_{l}}{\rho_{p}} E, \\
W_{1}=\frac{\sigma_{e}}{\rho_{l}} \frac{\sigma_{l}}{\rho_{p}} \frac{\sigma_{p}}{\rho_{w}} \frac{1}{\left[1+\frac{\gamma_{h} \varepsilon \beta_{w}^{\prime}}{\rho_{w} \rho_{i}} h\right]} E, \\
W_{2}=\frac{\sigma_{e}}{\rho_{l}} \frac{\sigma_{l}}{\rho_{p}} \frac{\sigma_{p}}{\rho_{w} \varepsilon \beta_{w}^{\prime}} \frac{\gamma_{w} \rho_{i}\left[1+\frac{\gamma_{h} \varepsilon \beta_{w}^{\prime}}{\rho_{w} \rho_{i}} h\right]}{\rho_{2}} h E, \\
W_{3}=\frac{\gamma_{w}}{\rho_{3}} W_{2}, \\
s=\frac{1}{R}\left[1+\frac{\gamma_{h} \varepsilon \beta_{w}^{\prime}\left(\rho_{3}+\gamma_{w}\right)}{\rho_{2} \rho_{3} \rho_{i}} h\right] \\
i=\frac{\gamma_{h}}{\rho_{i}} h \\
r=\frac{\gamma_{h}}{\rho_{i}} \frac{\sigma_{h}}{\mu_{h}} h,
\end{array}\right.
$$

mais os valores para $E$ e $h$ dados por

$$
\left\{\begin{array}{l}
E=C^{\prime}\left(1-\frac{\phi^{\prime}}{\phi}\right), \\
h=\frac{\gamma_{w} \mu_{h} \varepsilon \beta_{h}^{\prime}}{\rho_{h}\left[\mu_{h}\left(\rho_{3}+\gamma_{w}\right)+\gamma_{w} \varepsilon \beta_{h}^{\prime}\right]}\left(1-\frac{1}{R}\right),
\end{array}\right.
$$

que são válidos, biologicamente, para $\phi>\phi^{\prime}$ e $R>1$, onde $\phi^{\prime}$ é dada por

$$
\phi^{\prime}=\frac{\phi_{t h}}{\left[1+\frac{\gamma_{h} \varepsilon \beta_{w}^{\prime}\left(\rho_{3}+\gamma_{w}\right)}{\rho_{2} \rho_{3} \rho_{i}} h\right] \frac{\rho_{w} \rho_{i}}{\gamma_{h} \varepsilon \beta_{w}^{\prime} h+\rho_{w} \rho_{i}}} .
$$

Quando não houver transmissão da dengue $(h=0)$, tem-se $\phi^{\prime}=\phi_{t h}$. A função $\phi^{\prime}$ é estritamente crescente com $h$, com

$$
\phi_{\infty}=\frac{\rho_{e} \rho_{l} \rho_{p} \rho_{2} \rho_{3}}{\sigma_{e} \sigma_{l} \sigma_{p}\left(\gamma_{w}+\rho_{3}\right)},
$$

sendo o valor assintótico (matematicamente, pois biologicamente $h \rightarrow 1$ ) obtido no limite $h \rightarrow \infty$. 
A razão de reprodutibilidade $R$ dada pela equação (3.4) tem a seguinte interpretação biológica. A probabilidade de um mosquito (ou homem) sobreviver durante toda a fase de incubação extrínseca (ou latente) e tornar mosquito (ou homem) infectante é dada por $\gamma_{w} / \rho_{2}$ (ou $\left.\gamma_{h} / \rho_{h}\right) ; \varepsilon \beta_{h}^{\prime} / \rho_{3}$ é o número médio de indivíduos suscetíveis picados por um mosquito infectante durante todo o seu período infeccioso; e $\varepsilon \beta_{w}^{\prime} / \rho_{i}$ é o número médio de mosquitos suscetíveis que picam um indivíduo infectante durante todo o seu período infeccioso. Logo o produto dos quatro termos da equação (3.4) é o número médio de indivíduos suscetíveis picados por um mosquito infectante durante todo o seu período infeccioso $\left(\varepsilon \beta_{h}^{\prime} / \rho_{3}\right)$, que sobrevivem todo o período de incubação e tornam-se indivíduos infectantes $\left(\gamma_{h} / \rho_{h}\right)$, e que, por sua vez, são picados por mosquitos suscetíveis durante todo o período infeccioso destes indivíduos $\left(\varepsilon \beta_{w}^{\prime} / \rho_{i}\right)$ que sobrevivem o período de incubação extrínseca e tornam-se mosquitos infectantes $\left(\gamma_{w} / \rho_{2}\right)$. A interpretação do resultado matemático $R$ mostra que a infecção transmite-se quando um mosquito suscetível picar sucessivamente um indivíduo infectante e, posteriormente, um suscetível. Assim, quando não houver controle, $R_{0}$ é o número médio de mosquitos infectantes secundários produzidos por um único mosquito infectante durante todo o período infeccioso em populações homogêneas, sendo pior a epidemia quanto maior for o seu valor.

A estabilidade do ponto de equilíbrio trivial não se faz por meio da equação característica, pois na matriz Jacobiana (do sistema dinâmico) tem-se divisão por zero [5]. Por isso, limita-se apenas à análise de estabilidade do ponto de equilíbrio da população humana infestada de mosquitos sem dengue, dada pela equação (3.6). A equação característica correspondente ao sistema linearizado em torno de equilíbrio (que tem dois auto-valores idênticos $-\mu_{h}$ ) é dada por

$$
\Lambda(\lambda)=P_{1}(\lambda) P_{2}(\lambda)=0,
$$

onde os polinômios de quarto grau $P_{1}(\lambda)$ e $P_{2}(\lambda)$ são dados por

$$
\left\{\begin{array}{l}
P_{1}(\lambda)=\left(\rho_{e} R_{0}+\lambda\right)\left(\rho_{l}+\lambda\right)\left(\rho_{p}+\lambda\right)\left(\rho_{w}+\lambda\right)-\phi_{t h} \sigma_{e} \sigma_{l} \sigma_{p}=0, \\
P_{2}(\lambda)=\left(\rho_{2}+\lambda\right)\left(\rho_{3}+\lambda\right)\left(\rho_{h}+\lambda\right)\left(\rho_{i}+\lambda\right)-\varepsilon^{2} \gamma_{w} \gamma_{h} \beta_{w} \beta_{h}=0
\end{array}\right.
$$

e os termos independentes correspondentes de $\lambda$ são dados, respectivamente, por

$$
\left\{\begin{array}{l}
a_{1}^{0}=\rho_{e} \rho_{l} \rho_{p} \rho_{w}\left(\frac{\phi}{\phi_{t h}}-1\right) \\
a_{2}^{0}=\rho_{2} \rho_{3} \rho_{h} \rho_{i}(1-R) .
\end{array}\right.
$$

Note que os termos independentes são positivos para $\phi>\phi_{t h}$ e $R<1$. Assim, o ponto de equilíbrio não-trivial dado pela equação (3.6) é localmente e assintoticamente estável se $\phi>\phi_{t h}$ e $R<1$ [2]. Dessa forma, conjectura-se (de resultados numéricos) que, se $\phi<\phi_{t h}$ e $R<1$, então o equilíbrio trivial é estável, enquanto que, se $\phi>\phi_{t h}$ e $R>1$, então o equilíbrio endêmico da dengue é estável.

Para a dinâmica da transmissão da dengue, $\operatorname{com} \varphi(W)=\phi \sqrt{W}$ e $\varphi(W)=\phi W^{2}$, a única equação no estado estacionário que difere da equação (3.7) é o compartimento de ovos. Assim, basta resolver o sistema em equilíbrio com a equação

$$
\varphi(W)\left[1-\frac{E(t)}{C^{\prime}}\right]-\rho_{e} E(t)=0
$$


com uma das funções $\varphi(W)=\phi \sqrt{W}$ e $\varphi(W)=\phi W^{2}$.

\subsection{Encontro das massas}

Estuda-se o caso do encontro das massas, com as forças de infecções dadas pela equação (3.2). Os pontos de equilíbrio dos sistemas de equações (2.1) e (2.3) com as forças de infecção dadas pela equação (3.2) são três.

População humana livre de mosquitos. Os valores para cada compartimento de mosquitos e homens são os mesmos dados pela equação (3.3), que são válidos, biologicamente, para $\phi<\phi_{t h}$. Este é o equilíbrio trivial.

População humana infestada por mosquitos sem a transmissão da dengue. Os valores para cada compartimento de mosquitos e homens são os mesmos dados pela equação (3.6), cujos valores são válidos, biologicamente, para $\phi>\phi_{t h}$ e $R^{\prime}=$ $E R_{n} \rho_{e} / \phi_{t h}<1$, ou,

$$
R^{\prime}=R_{n} \frac{\rho_{e}}{\phi_{t h}}\left[C^{\prime}\left(1-\phi_{t h} / \phi\right)\right],
$$

onde $\phi_{t h}$ é dada pela equação (3.5) e $R_{n}$, pela equação (3.4) trocando-se $\beta_{h}^{\prime}$ por $\beta_{h}$. O parâmetro $R^{\prime}$ representa a razão de reprodutibilidade da dengue, pois está-se considerando o controle do vetor. Quando não houver controle, tem-se, então, a razão de reprodutibilidade basal $R_{0}^{\prime}$.

População humana infestada por mosquitos com a transmissão da dengue. Os valores para cada compartimento de mosquitos e homens são os mesmos dados pela equação (3.7), trocando-se $\beta_{h}^{\prime}$ por $\beta_{h}$, exceto para $s$, e os valores para $E$ (solução de uma equação de segundo grau) e $h$ dados por

$$
\left\{\begin{array}{l}
s=\frac{1}{R}\left[1+\frac{\gamma_{h} \varepsilon \beta_{w}^{\prime}}{\rho_{w} \rho_{i}} h\right], \\
a_{2} E^{2}+a_{1} E+a_{0}=0, \\
h=\frac{\mu_{h} q \phi_{t h}}{\rho_{h} \rho_{e} R_{n}} \times \frac{R^{\prime}-1}{E q+\mu_{h}},
\end{array}\right.
$$

que são válidos, biologicamente, para $R^{\prime}=E R_{n} \rho_{e} / \phi_{t h}>1$, onde a variável $q$ é dada por

$$
q=\frac{\gamma_{w} \varepsilon \beta_{h} \sigma_{e} \sigma_{l} \sigma_{p}}{\rho_{2} \rho_{3} \rho_{p} \rho_{l}}
$$

e os coeficientes são dados por

$$
\left\{\begin{array}{l}
a_{0}=-\frac{\gamma_{w} \mu_{3}+\rho_{3} \mu_{2}}{\rho_{2} \rho_{3}}, \\
a_{1}=\frac{\gamma_{w} \mu_{3}+\rho_{3} \mu_{2}}{C^{\prime} \rho_{2} \rho_{3}}-\frac{q}{\mu_{h}}\left\{1-\frac{1}{Q}+\frac{\gamma_{h} \varepsilon \beta_{w}^{\prime} \mu_{h}}{\rho_{w} \rho_{h} \rho_{i}}\left[\frac{\left(\rho_{3}+\gamma_{w}\right) \rho_{w}}{\rho_{2} \rho_{3}}-\frac{1}{Q}\right]\right\}, \\
a_{2}=\frac{q}{C^{\prime} \mu_{h}}\left[1+\frac{\gamma_{h} \varepsilon \beta_{w}^{\prime}\left(\rho_{3}+\gamma_{w}\right) \mu_{h}}{\rho_{2} \rho_{3} \rho_{h} \rho_{i}}\right] .
\end{array}\right.
$$

Se $R^{\prime}>1$, então a condição $\phi>\phi_{t h}$ é satisfeita automaticamente. A equação de segundo grau tem uma única solução real positiva, pois $a_{0}<0$ e $a_{2}>0$.

A razão de reprodutibilidade $R^{\prime}$ (ausência da dengue, $h=0$ ) dada pela equação (3.11) depende de $R_{n}$, da razão $\rho_{e} / \phi_{t h}$ e da capacidade remanescente $C^{\prime}$, pois a transmissão depende da quantidade de mosquitos adultos infectantes, e não da 
fração deles. Assim, as possibilidades de erradicação (tornando $R^{\prime}$ menor que 1) da dengue podem ser obtidas, além de atuar para diminuir $R_{n}$, quando se diminui o valor de $\rho_{e}$ (impedindo que ovos cheguem ao estágio de mosquito adulto infectante) e/ou aumentando $\phi_{t h}$ (aumentando a dificuldade para a perpetuação dos mosquitos ao elevar o valor crítico para a taxa de oviposição) e/ou reduzindo número de criadouros $C^{\prime}$ (intensas campanhas educativas).

A análise de estabilidade do ponto de equilíbrio da população humana livre de mosquitos é feita pela equação característica (3.10), que tem dois auto-valores idênticos $-\mu_{h}$, onde os polinômios de quarto grau $P_{1}(\lambda)$ e $P_{2}(\lambda)$ são dados por

$$
\left\{\begin{array}{l}
P_{1}(\lambda)=\left(\rho_{e}+\lambda\right)\left(\rho_{l}+\lambda\right)\left(\rho_{p}+\lambda\right)\left(\rho_{w}+\lambda\right)-\phi \sigma_{e} \sigma_{l} \sigma_{p}=0 \\
P_{2}(\lambda)=\left(\rho_{2}+\lambda\right)\left(\rho_{3}+\lambda\right)\left(\rho_{h}+\lambda\right)\left(\rho_{i}+\lambda\right)=0
\end{array}\right.
$$

e os termos independentes correspondentes de $\lambda$ são dados, respectivamente, por

$$
\left\{\begin{array}{l}
a_{1}^{0}=\rho_{e} \rho_{l} \rho_{p} \rho_{w}\left(1-\frac{\phi}{\phi_{t h}}\right) \\
a_{2}^{0}=\rho_{2} \rho_{3} \rho_{h} \rho_{i} .
\end{array}\right.
$$

Note que o termo independente para $P_{1}(\lambda)$ é positivo para $\phi<\phi_{t h}$. Assim, o ponto de equilíbrio trivial é localmente e assintoticamente estável se $\phi<\phi_{t h}[2]$, pois todos os auto-valores do polinômio $P_{2}(\lambda)$, dados por $\lambda_{1}=-\rho_{2}, \lambda_{2}=-\rho_{3}, \lambda_{3}=-\rho_{h}$ e $\lambda_{4}=-\rho_{i}$, são reais negativos.

A análise de estabilidade do ponto de equilíbrio da população humana infestada de mosquitos sem dengue é feita pela equação característica (3.10), que tem dois auto-valores idênticos $-\mu_{h}$, onde os polinômios de quarto grau $P_{1}(\lambda)$ e $P_{2}(\lambda)$ são

$$
\left\{\begin{array}{l}
P_{1}(\lambda)=\left(\rho_{e} R_{0}+\lambda\right)\left(\rho_{l}+\lambda\right)\left(\rho_{p}+\lambda\right)\left(\rho_{w}+\lambda\right)-\phi_{t h} \sigma_{e} \sigma_{l} \sigma_{p}=0, \\
P_{2}(\lambda)=\left(\rho_{2}+\lambda\right)\left(\rho_{3}+\lambda\right)\left(\rho_{h}+\lambda\right)\left(\rho_{i}+\lambda\right)-\varepsilon^{2} \gamma_{w} \gamma_{h} \beta_{w}^{\prime} \beta_{h} \frac{\sigma_{e} \sigma_{l} \sigma_{p}}{\rho_{l} \rho_{p} \rho_{w}} E=0
\end{array}\right.
$$

e os termos independentes correspondentes de $\lambda$ são dados, respectivamente, por

$$
\left\{\begin{array}{l}
a_{1}^{0}=\rho_{e} \rho_{l} \rho_{p} \rho_{w}\left(\frac{\phi}{\phi_{t h}}-1\right) \\
a_{2}^{0}=\rho_{2} \rho_{3} \rho_{h} \rho_{i}\left(1-R^{\prime}\right) .
\end{array}\right.
$$

Note que os termos independentes são positivos para $\phi>\phi_{t h}$ e $R^{\prime}<1$. Assim, o ponto de equilíbrio não-trivial sem dengue é localmente e assintoticamente estável se $\phi>\phi_{t h}$ e $R^{\prime}<1$ [2]. Conjectura-se (de resultados numéricos) que, se $\phi>\phi_{t h}$ e $R^{\prime}<1$ então o equilíbrio não-trivial (sem dengue) é estável, enquanto que, se $\phi>\phi_{t h}$ e $R^{\prime}>1$, então o equilíbrio endêmico da dengue é estável [2]. Quando a dengue é endêmica, $R^{\prime}$ depende de $h$ via $E$ e não é mais uma constante como $R$.

As duas diferentes definições para as forças de infecção diferem apenas quando a dengue é endêmica na comunidade. Este fato surge devido à diminuição do número de mosquitos infectantes por causa do envelhecimento.

\section{Conclusão}

A propagação da dengue foi estudada através de modelo matemático. Os resultados analíticos obtidos do modelo consideraram os parâmetros constantes no tempo. 
Quando se considera no modelo a infecção como um evento probabilístico (ou "verdadeira" lei da ação das massas, segundo alguns autores), seus resultados diferem do modelo em que a infecção propaga-se de acordo com o encontro das massas ("falsa" lei da ação das massas, segundo alguns autores). Porém, esta diferenciação só aparece quando se trata de população total variando no tempo (no caso, os mosquitos), enquanto no caso de população constante no tempo, os resultados epidemiológicos são os mesmos (no caso da população humana).

A erradicação da dengue pode ser obtida diminuindo-se a razão de reprodutibilidade $R$ (transmissão ocorrendo como evento probabilístico, ou $R^{\prime}$, quando do encontro das massas) para valores abaixo da unidade. Assim, a doença pode ser erradicada da população humana mesmo que não ocorra eliminação total dos mosquitos. No entanto, a razão de reprodutibilidade basal é a mesma para os dois modelos, dada por $R_{0}$. As bruscas variações da incidência de dengue em função das condições abióticas são estudadas usando-se modelos matemáticos com sistemas de equações não-autônomos [1], cujos resultados são melhor compreendidos e interpretados levando-se em consideração os resultados analíticos aqui obtidos.

Abstract. The dynamics of dengue transmission is assessed by mathematical models. The models consider two interacting populations, in order to describe the overall transmission of dengue virus by mosquitos Aedes aegypti to humans.

\section{Referências}

[1] C.P. Ferreira e H.M. Yang, Estudo da transmissão da dengue entre os indivíduos em interação com a população de mosquitos Aedes aegypti, em "Seleta do XXV CNMAC" (E.X.L. de Andrade et al., eds.), TEMA Tend. Mat. Apl. Comput., 4, No. 2 (2003), no prelo.

[2] M.B.F. Leite, R.C. Bassanezi e H.M. Yang, The basic reproduction ratio for a model of directly transmitted infections considering the virus charge and the immunological response, IMA J. Math. Appl. Med. Biol., 17, No. 1 (2000), 1531.

[3] R. Veronesi, "Doenças Infecciosas e Parasitárias", Oitava Edição, Guanabara Koogan, Rio de Janeiro, 1991.

[4] H.M. Yang, "Epidemiologia Matemática - Estudo dos Efeitos da Vacinação em Doneças de Transmissão Direta", EDUNICAMP e FAPESP, Campinas e São Paulo, 2001.

[5] H.M. Yang, C.P. Ferreira e S. Ternes, Dinâmica populacional do vetor transmissor da dengue, em "Seleta do XXV CNMAC" (E.X.L. de Andrade et al., eds.), TEMA Tend. Mat. Apl. Comput., 4, No. 2 (2003), no prelo. 